\title{
Compatible Mapping and Common Fixed Point Theorem
}

\author{
Rajesh Shrivastava ${ }^{1}$, Neha Jain ${ }^{2}$, K. Qureshi ${ }^{3}$ \\ ${ }^{I}$ Deptt . of Mathematics, Govt. Science and comm. College Benazir Bhopal( M.P) India \\ ${ }^{2}$ Research Scholar, Govt. Science and comm. College Benazir Bhopal (M.P)" \\ ${ }^{3}$ Additional Director, Higher Education Deptt. Govt. of M.P., Bhopal (M.P) India
}

Abstract: In this paper we prove common fixed point theorem for compatible mapping.

Key Words: Common Fixed Point, Compatible Mapping ,Commuting mapping, Metric Space

\section{Introduction}

The study of Common Fixed Point of mapping satisfying contraction type condition has been a very active field of research activity during the last three decades. The concept of common fixed point theorem for commuting mappings was given by Jungck [6], which generalizes the Banach's [1] Fixed Point Theorem. The result Was generalized and extended in various ways by Iseki and singh [5],Park [12],Das And Naik [2],Singh [15]Singh and Singh [16],Fisher [3],Park and Bae [13]. Recently, some Common Fixed Point Theorems of three and four commuting mappings were proved by Fisher [3],Khan and Imdad [10], Kang and kim [9],and Lohani and Badshah [11].

The concept of generalizion of commutability is given by Seesa[14], which is called weak commutability, which generalizes the result of Das and Naik [2],More generalized commutability was introduced by Jungck [7],which is called compatibility .The utility of compatibility was initially demonstrated in extending a theorem of Park and Bae [13 ] in the concept of Fixed Point Theory. In general, commuting mappings are weakly commuting and weakly commuting mapping are compatible, but the converse are not necessarily true. The purpose of this paper is to generalize a common Fixed Point Theorem, which extend the result of Fisher[4], Jungck [8], and Lohani and Badshah [11] by using a functional inequality and compatible mappings instead of commuting mappings. To illustrate our main theorem,

\section{Preliminaries}

DEFINITIONS 2.1: - If $S$ and $T$ are mappings from a metric space $(X, d)$ into itself , are called

(i) Commuting on $\mathrm{X}$ if $\mathrm{d}(\mathrm{ST} x, \mathrm{TSx})=0$ for all $\mathrm{x}$ in $\mathrm{X}$.

(ii) Weakly commuting on $\mathrm{X}$, if

$\mathrm{d}(\mathrm{ST}, \mathrm{TSx}) \leq \mathrm{d}(\mathrm{Sx}, \mathrm{Tx})$ for all $\mathrm{x}$ in $\mathrm{X}$. Commuting mappings are weakly commuting, but he converse is not necessarily true.

DEFINITION 2.2:-If $\mathrm{S}$ and $\mathrm{T}$ are mapping from a metric space $(\mathrm{X}$,d) into itself , are called compatible on $\mathrm{X}$.if $\operatorname{limSx_{m}}=\operatorname{limTx}_{\mathrm{m}}=\mathrm{x}$ for some point $\mathrm{x}$ in $\mathrm{X}$.

$\mathrm{m} \rightarrow \infty \quad \mathrm{m} \rightarrow \infty$

clearly, $\mathrm{S}$ and $\mathrm{T}$ are compatible mappings on $\mathrm{X}$, then $\mathrm{d}(\mathrm{STx}, \mathrm{TSx})=0$ when $\mathrm{d}(\mathrm{Sx}, \mathrm{Tx})=0$ for some $\mathrm{x}$ in $\mathrm{X}$.

Weakly commuting mappings are compatible; the converse is not necessarily true:

Lemma 2.1[7]:- Let $S$ and $T$ be compatible mappings from a Metric space $(X, d)$ into itself. Suppose that $\lim \mathrm{Sx}_{\mathrm{m}}=\lim \mathrm{Tx}_{\mathrm{m}}=\mathrm{x}$ for some point $\mathrm{x}$ in $\mathrm{X}$.

$\mathrm{m} \rightarrow \infty \quad \mathrm{m} \rightarrow \infty$

Then $\lim \mathrm{Tx}_{\mathrm{m}}=\mathrm{S} \mathrm{x}$, if $\mathrm{S}$ is continuous $\mathrm{m} \rightarrow \infty$

$$
\mathrm{m} \rightarrow \infty
$$

Now Let $\mathrm{P}, \mathrm{Q}, \mathrm{S}$ and $\mathrm{T}$ are mappings from a complete Metric space $(\mathrm{X}, \mathrm{d})$ into itself satisfying the condition

$\begin{aligned} & S(X) \subset Q(X), T(X) \subset P(X) \\ d(S x, T y) \leq \quad & \alpha\left\{[d(P x, S x)]^{3}+[d(Q y, T y)]^{3} /[d(P x, S x)]^{2}+[d(Q y, T y)]^{2}\right\}+\beta d(P x, Q y)\end{aligned}$

For all $\mathrm{x}, \mathrm{y} \in \mathrm{X}$, where $\alpha, \beta \geq 0$ and $\alpha+\beta<1$. Then for an arbitrary point $\mathrm{x}_{0} \in \mathrm{X}$, by (A) we choose a point $\mathrm{x}_{1}$ in $\mathrm{X}$ such that

$\mathrm{Qx}_{1}=\mathrm{Sx}_{0}$ and for this point $\mathrm{x}_{1}$, there exist a point $\mathrm{x}_{2}$ in $\mathrm{X}$ such that

$\mathrm{Px}_{2}=\mathrm{Tx}_{1}$ and so on. Proceeding in the similar manner, we can define a sequence $\left\{\mathrm{y}_{\mathrm{m}}\right\}$ in $\mathrm{X}$ such that

$\mathrm{Y}_{2 \mathrm{~m}+1}=\mathrm{Q} \mathrm{x}_{2 \mathrm{~m}+1}=\mathrm{Sx}_{2 \mathrm{~m}}$ 
And

$\mathrm{Y}_{2 \mathrm{~m}}=\mathrm{Px}_{2 \mathrm{~m}}=\mathrm{Tx}_{2 \mathrm{~m}-1}$

Lemma 2.2[8] :- Let $\mathrm{P}, \mathrm{Q}, \mathrm{S}$, and $\mathrm{T}$ be mappings from a Metric Space $(\mathrm{X}, \mathrm{d})$ into itself satisfying the conditions (A) and (B) . then the sequence $\left\{\mathrm{y}_{\mathrm{m}}\right\}$ defined by $(C)$ is a Cauchy sequence .

\section{Our aim of this paper to prove the following theorem:-}

\section{Main Result}

Theorem: - Let P, Q, S and T be mapping s from a complete Metric Space (X ,d) into itself satisfying the conditions

(i) $\quad \mathrm{P}(\mathrm{X}) \subset \mathrm{T}(\mathrm{X}), \mathrm{Q}(\mathrm{X}) \subset \mathrm{S}(\mathrm{X})$

(ii) $\quad[\mathrm{d}(\mathrm{Px}, \mathrm{Qy})]^{2} \leq \mathrm{a}[\mathrm{d}(\mathrm{Px}, \mathrm{Sx}) \mathrm{d}(\mathrm{Qy}, \mathrm{Ty})+\mathrm{d}(\mathrm{Qy}, \mathrm{Sx}) \mathrm{d}(\mathrm{Px}, \mathrm{Ty})]+$ where $0 \leq a+2 b<1 ; a, b \geq 0$ $\mathrm{b}[\mathrm{d}(\mathrm{Px}, \mathrm{Sx}) \mathrm{d}(\mathrm{Px}, \mathrm{Ty})+\mathrm{d}(\mathrm{Qy}, \mathrm{Ty}) \mathrm{d}(\mathrm{Qy}, \mathrm{Sx})]$

(a) One of $\mathrm{P}, \mathrm{Q}, \mathrm{S}$ and $\mathrm{T}$ is continuous .

(b) $\mathrm{P}, \mathrm{S}$ and $\mathrm{Q}, \mathrm{T}$ are compatible on $\mathrm{X}$.

Then $\mathrm{P}, \mathrm{Q}, \mathrm{S}$ and $\mathrm{T}$ have a unique common fixed point in $\mathrm{X}$.

PROOF :- $\quad$ Let $\left\{\mathrm{y}_{\mathrm{n}}\right\}$ is Cauchy sequence and since $\mathrm{X}$ is complete so there exist a point $\mathrm{z} \& \mathrm{X}$ such that $\lim \mathrm{y}_{\mathrm{n}}=\mathrm{z}$ as $\mathrm{n} \rightarrow \infty$.

consequently sequences $\mathrm{Px}_{2 \mathrm{n}}, \mathrm{Sx}_{2 \mathrm{n}}, \mathrm{Qx}_{2 \mathrm{n}-1}$ and $\mathrm{Tx}_{2 \mathrm{n}+1}$ converges to $\mathrm{z}$.

Let $S$ be continuous. Since $P$ and $S$ are compatible on $X$. we have $\mathrm{S}^{2} \mathrm{x}_{2 n} \rightarrow \mathrm{Sz}$ and $\mathrm{PSx}_{2 \mathrm{n}} \rightarrow \mathrm{Sz}$ as $\mathrm{n} \rightarrow \infty$, $[d(P x, Q y)]^{2} \leq a[d(P x, S x) d(Q y, T y)+d(Q y, S x) d(P x, T y)]+b[d(P x, S x) d(P x, T y)+d(Q y, T y) d(Q y, S x)]$ we have

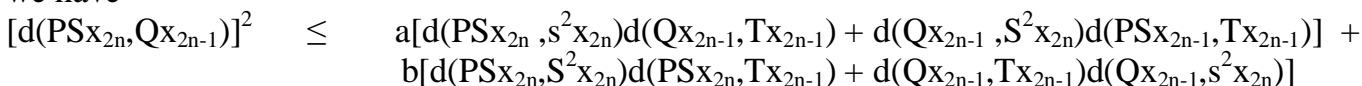

as $\mathrm{n} \rightarrow \infty$

we have $[\mathrm{d}(\mathrm{Sz}, \mathrm{z})]^{2} \leq \mathrm{a}[\mathrm{d}(\mathrm{Sz}, \mathrm{z})]^{2}$

Which is a contradiction .

Hence $\mathrm{Sz}=\mathrm{z}$

Now

$\left[\mathrm{d}\left(\mathrm{Pz}, \mathrm{Qx}_{2 \mathrm{n}-1}\right)\right]^{2} \leq \mathrm{a}\left[\mathrm{d}(\mathrm{Pz}, \mathrm{Sz}) \mathrm{d}\left(\mathrm{Qx}_{2 \mathrm{n}-1}, \mathrm{Tx}_{2 \mathrm{n}-1}\right)+\mathrm{d}\left(\mathrm{Qx}_{2 \mathrm{n}-1}, \mathrm{Sz}\right) \mathrm{d}\left(\mathrm{Pz}, \mathrm{Tx}_{2 \mathrm{n}-1}\right)\right]+$

Letting $\mathrm{n} \rightarrow \infty$, we have $\mathrm{b}\left[\mathrm{d}(\mathrm{Pz}, \mathrm{Sz}) \mathrm{d}\left(\mathrm{Pz}_{2}, \mathrm{Tx}_{2 \mathrm{n}-1}\right)+\mathrm{d}\left(\mathrm{Qx}_{2 \mathrm{n}-1}, \mathrm{Tx}_{2 \mathrm{n}-1}\right) \mathrm{d}\left(\mathrm{Qx}_{2 \mathrm{n}-1}, \mathrm{Sz}\right)\right]$

$[\mathrm{d}(\mathrm{Pz}, \mathrm{z})]^{2} \leq \mathrm{a}[\mathrm{d}(\mathrm{Pz}, \mathrm{z}) \mathrm{d}(\mathrm{z}, \mathrm{z})+\mathrm{d}(\mathrm{z}, \mathrm{Sz}) \mathrm{d}(\mathrm{Pz}, \mathrm{z})]+\mathrm{b}[\mathrm{d}(\mathrm{Pz}, \mathrm{z}) \mathrm{d}(\mathrm{Pz}, \mathrm{z})+\mathrm{d}(\mathrm{z}, \mathrm{z}) \mathrm{d}(\mathrm{z}, \mathrm{z})]$

$\leq \mathrm{a}[\mathrm{d}(\mathrm{Pz}, \mathrm{z}) \mathrm{d}(\mathrm{z}, \mathrm{z})+\mathrm{d}(\mathrm{z}, \mathrm{z}) \mathrm{d}(\mathrm{P} \mathrm{z}, \mathrm{z})]+\mathrm{b}[\mathrm{d}(\mathrm{Pz}, \mathrm{z}) \mathrm{d}(\mathrm{Pz}, \mathrm{z})+\mathrm{d}(\mathrm{z}, \mathrm{z}) \mathrm{d}(\mathrm{z}, \mathrm{z})]$

$\left.\leq \mathrm{a}[\mathrm{d}(\mathrm{Pz}, \mathrm{z}) 0+0 \mathrm{~d}(\mathrm{Pz}, \mathrm{z})]+\mathrm{b}[\mathrm{d}(\mathrm{Pz}, \mathrm{z})]^{2}+0\right]$

$\leq \mathrm{a}[0+0]+\mathrm{b}[\mathrm{d}(\mathrm{Pz}, \mathrm{z})]^{2}$

$[\mathrm{d}(\mathrm{Pz}, \mathrm{z})]^{2} \leq \mathrm{b}[\mathrm{d}(\mathrm{Pz}, \mathrm{z})]^{2}$

Which is a contradiction

Hence $\mathrm{Pz}=\mathrm{z}$

Now since $\mathrm{Pz}=\mathrm{z}$ by condition (i) $\mathrm{z} \varepsilon \mathrm{T}(\mathrm{X})$.

Also $T$ is self map of $X$. so there exist a point $u \varepsilon X$ such that $z=P z=T u$. More over by condition (ii) we obtain

$[\mathrm{d}(\mathrm{z}, \mathrm{Qu})]^{2}=[\mathrm{d}(\mathrm{Pz}, \mathrm{Qu})]^{2} \leq \mathrm{a}[\mathrm{d}(\mathrm{Pz}, \mathrm{Sz}) \mathrm{d}(\mathrm{Qu}, \mathrm{Tu})+\mathrm{d}(\mathrm{Qu}, \mathrm{Sz}) \mathrm{d}(\mathrm{Pz}, \mathrm{Tu})]+$

i.e $\mathrm{b}[\mathrm{d}(\mathrm{Pz}, \mathrm{Sz}) \mathrm{d}(\mathrm{Pz}, \mathrm{Tu})+\mathrm{d}(\mathrm{Qu}, \mathrm{Tu}) \mathrm{d}(\mathrm{Qu}, \mathrm{Sz})]$

$[\mathrm{d}(\mathrm{z}, \mathrm{Qu})]^{2} \leq \mathrm{b}[\mathrm{d}(\mathrm{z}, \mathrm{Qu})]^{2}$

Hence $\quad \mathrm{Qu}=\mathrm{z}$ i.e $\mathrm{z}=\mathrm{Tu}=\mathrm{Qu}$

We have $\mathrm{TQu}=\mathrm{QTu} \quad[$ by the definition of the compatible]

Hence $\mathrm{Tz}=\mathrm{Qz}$

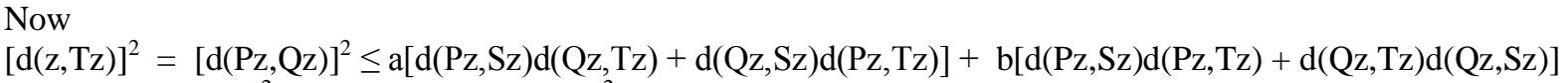

i.e $\left.\left.[\mathrm{d}(\mathrm{z}, \mathrm{Tz})]^{2} \leq \mathrm{az}\right)\right]^{2} \leq \mathrm{a}\left[\mathrm{d}(\mathrm{Pz}, \mathrm{Sz}(\mathrm{z}, \mathrm{Tz})]^{2}\right.$

which is a contradiction. Hence $\mathrm{z}=\mathrm{Tz}$ i.e $\mathrm{z}=\mathrm{Tz}=\mathrm{Qz}$ 
Therefore $\mathrm{z}$ is common fixed point of $\mathrm{P}, \mathrm{Q}, \mathrm{S}$ and $\mathrm{T}$.

Similarly we can prove this when any one of $\mathrm{P}, \mathrm{Q}$ or $\mathrm{T}$ is continuous.

Finally, in order to prove the uniqueness of $\mathrm{z}$, suppose $\mathrm{w}$ be another common

Fixed Point P, Q, S and T

then we have

$[\mathrm{d}(\mathrm{z}, \mathrm{w})]^{2}=[\mathrm{d}(\mathrm{Pz}, \mathrm{Qw})]^{2} \leq \mathrm{a}[\mathrm{d}(\mathrm{Pz}, \mathrm{Sz}) \mathrm{d}(\mathrm{Qw}, \mathrm{Tw})+\mathrm{d}(\mathrm{Qw}, \mathrm{Sw}) \mathrm{d}(\mathrm{Pz}, \mathrm{Tw})]+$

which gives $\mathrm{b}[\mathrm{d}(\mathrm{Pz}, \mathrm{Sz}) \mathrm{d}(\mathrm{Pz}, \mathrm{Tw})+\mathrm{d}(\mathrm{Qw}, \mathrm{Tw}) \mathrm{d}(\mathrm{Qw}, \mathrm{Sz})]$

$[\mathrm{d}(\mathrm{z}, \mathrm{Tw})]^{2} \leq \mathrm{a}[\mathrm{d}(\mathrm{z}, \mathrm{Tw})]^{2}$. Hence $\mathrm{z}=\mathrm{w}$

This completes the proof. Therefore, $z$ is a unique common Fixed Point of $P, Q, S$ and $T$.

Hence proved......

COROLLARY3.1 :- $\quad$ Let $P, Q, S$ and $T$ be mapping s from a complete Metric Space (X,d) into itself satisfying the conditions

$$
\mathrm{P}(\mathrm{X}) \subseteq \mathrm{T}(\mathrm{X}), \mathrm{Q}(\mathrm{X}) \subseteq \mathrm{S}(\mathrm{X})
$$

(ii) $\quad[d(P x, Q y)]^{2} \leq a[d(P x, S x) d(Q y, T y)+d(Q y, S x) d(P x, T y)]+$

where $0 \leq a+2 b<1 ; a, b \geq 0$

$$
\mathrm{b}[\mathrm{d}(\mathrm{Px}, \mathrm{Sx}) \mathrm{d}(\mathrm{Px}, \mathrm{Ty})+\mathrm{d}(\mathrm{Qy}, \mathrm{Ty}) \mathrm{d}(\mathrm{Qy}, \mathrm{Sx})]
$$

Then $P, Q, S$ and $T$ have a unique common Fixed Point.

\section{Refrences}

[1] Banach , S., Sur les operations dans les ensembles abstraits et

[2] Das, K.M. and Naik, K.V., common Fixed point Theorems for

leurs applications ,Fund. Math. 3:133-181(1922). Math.Soc.77:369(1979).

[3] Fisher, B., common Fixed Point of commuting mappings, Bull. Inst. Math. Acad. Scinica , 9:399(1981).

[4] Fisher, B., common Fixed Point of four mappings, Bull. Inst.Math. Acad. Scinica ,11: 103(1983).

[5] Iseki, K. and Singh ,Bijendra ,On common Fixed point theorems of mappings Math. Sem. Notes, Kobe Univ., 2: 96(1974).

[6] Jungck, G., commuting maps and Fixed points Amer .Math. Monthly, 83: 261(1976).

[7] Jungck, G., compatible mappings and common Fixed points ,Internat. J. Math. and Math .Sci., 9: 771(1986).

[8] ungck, G., compatible mappings and common Fixed points (2), Internat. J. Math. and Math .Sci., 11: 285(1985).

[9] Kang, S.M. and Kim ,Y.P., Common Fixed point theorems, Math. Japonica, 37: 1037(1992).

[10] Khan ,M .S. and Imdad, M., Some common Fixed Point theorems, Glesnik Mat.18:(38), 321(1983).

[11] Lohani, P.C. and Badshah , V.H., Compatibles mappings and common Fixed point for four mappings, Bull . Cal. Math . Soc. , 90:301-308(1998)

[12] Park, S., Fixed points of f-contractive maps, Rocky Mountain J.

[13] Park, S. and Bae, J.S., Extensions of common Fixed point

Math. , 8:743(1977)

theorem of Mier and Keeler, Ark. Math.19:223(1981).

[14] Seesa, S., On a weak commutativity condition of mappings in Fixed point considerations, Publ. Inst. Math.32:(46), 149(1982).

[15] Singh, S.L., Applications of a common fixed point theorem ,Math. Sem. Notes, 6: 37(1978).

[16] Singh, S.L. and singh, S.P., A fixed point theorem, Indian J. Pure Appl. Math.11: 1584(1980). 